\title{
Local mild solutions to three-dimensional magnetohydrodynamic system in Morrey spaces
}

\author{
Zirong Zeng ${ }^{1}$
}

${ }^{1}$ Shanghai Jiao Tong University

October 8, 2020

\begin{abstract}
In this article, the Cauchy problem of three-dimensional (3-D) incompressible magnetohydrodynamic system with infinite energy initial data is investigated. Via some elaborate analysis of the time evolution of both the vorticity $\omega=: \mathrm{V} \times \mathrm{u}$ and the current density $\mathrm{j}=: \mathrm{Vxb}$, the local-in-time well-posedness of mild solutions with arbitrarily large initial data in Morrey spaces is established.
\end{abstract}

\section{Hosted file}

MHD in Morrey Space.pdf available at https://authorea.com/users/365406/articles/485512-localmild-solutions-to-three-dimensional-magnetohydrodynamic-system-in-morrey-spaces 